\title{
The Role of the Nonlinearity of the Stefan-Boltzmann Law on the Structure of Radiatively Forced Temperature Change
}

\author{
MatThew Henry And Timothy M. MerLis \\ McGill University, Montreal, Quebec, Canada
}

(Manuscript received 8 September 2017, in final form 11 October 2018)

\begin{abstract}
The Stefan-Boltzmann law governs the temperature dependence of the blackbody emission of radiation: $E=\sigma T^{4}$. A consequence of this nonlinearity is that a cold object needs a greater increase in temperature than a hot object in order to reach the same increase in radiation emitted. Therefore, this nonlinearity potentially has an impact on the structure of radiatively forced atmospheric temperature change in both the horizontal and vertical directions. For example, it has previously been argued to be a cause of polar amplification (PA) of surface air warming. Here, the role of this nonlinearity is investigated by 1) assessing the magnitude of its effect on PA compared to spatial variations in $\mathrm{CO}_{2}$ 's radiative forcing for Earth's atmosphere and 2) linearizing $\sigma T^{4}$ in a gray radiation atmospheric general circulation model (GCM) with an interactive hydrological cycle. Estimates for Earth's atmosphere show that the combination of the Planck feedback and forcing from $\mathrm{CO}_{2}$ would produce a tropically amplified warming if they were the only means of changing the Earth's energy balance. Contrary to expectations, climate change simulations with linearized radiation do not have reduced polar amplification of surface air warming relative to the standard GCM configuration. However, simulations with linearized radiation consistently show less warming in the upper troposphere and more warming in the lower troposphere across latitudes. The lapse rate feedbacks from pure radiative and radiative-convective configurations of the model are used to show that the "cold-altitudes-warm-more" effect of the $\sigma T^{4}$ nonlinearity carries across this model hierarchy.
\end{abstract}

\section{Introduction}

The change in atmospheric temperature due to radiative forcing is vertically and horizontally inhomogeneous. The zonal-mean warming is amplified near the surface at the poles and in the upper troposphere in the tropics. Polar amplification (PA) of surface air warming is a common feature of climate model projections (Manabe and Wetherald 1975; Pithan and Mauritsen 2014). Observations of the recent climate also show faster transient warming in the Arctic (IPCC 2013), while Antarctic amplification is delayed as a result of ocean heat uptake (Manabe et al. 1991). In the tropics, there is amplified warming in the upper troposphere relative to the surface in future climate projections that is comparable to that of a warmed moist adiabat (Santer et al. 2005).

There are several mechanisms that are thought to contribute to the pattern of temperature change, and the challenge is to understand the relative importance of

Corresponding author: Matthew Henry, matthew.henry@mail. mcgill.ca each mechanism and the interactions between them. The main contributors to the PA of surface air warming are thought to be the surface albedo feedback (Winton 2006; Graversen et al. 2014), an increase in poleward energy transport (Alexeev and Jackson 2013; Lee 2014; Merlis and Henry 2018), and a destabilizing polar lapse rate feedback (Graversen et al. 2014; Pithan and Mauritsen 2014; Payne et al. 2015). The role of cloud and water vapor feedbacks on the PA of surface air warming is uncertain. According to Pithan and Mauritsen (2014), the ensemble-mean cloud change has a small positive impact on PA and water vapor counteracts PA via their radiative feedbacks in comprehensive climate models. Winton (2006) found that the total longwave feedback (which includes cloud, water vapor, and temperature) contributes to PA. Finally, the top-of-atmosphere (TOA) net radiation response to a vertically invariant temperature change, the Planck feedback, can have spatial structure that tends to amplify polar warming. The structure arises from the nonlinearity of the temperature dependence of the blackbody emission $E$ of radiation given by the Stefan-Boltzmann law, $E=\sigma T^{4}$. 
For example, while comparing contributions from various feedbacks to polar amplification on an aquaplanet, Langen et al. (2012) found that the Planck and lapse rate feedbacks lead to a polar amplified temperature change, with the water vapor feedback destabilizing, thereby increasing the global-mean temperature change without altering its pattern. Feldl and Roe (2013b) used an aquaplanet slab model with a simple parameterization of the ice-albedo feedback to analyze contributions to the surface air temperature change. They found that replacing the locally defined Planck feedback by its global mean led to $20 \%$ less warming in the high latitudes and $20 \%$ more warming in the tropics. Pithan and Mauritsen (2014) diagnosed that the latitudinal variation of the Planck feedback contributes $1.7 \mathrm{~K}$ of warming toward an average Arctic warming of $11.1 \mathrm{~K}$ and cools the tropics by $0.5 \mathrm{~K}$ relative to an average warming of $4.5 \mathrm{~K}$, by analyzing perturbation TOA energy budgets of models from phase 5 of the Coupled Model Intercomparison Project (CMIP5). Thus, while all of these studies find that the Planck feedback contributes to polar amplification, the magnitude of this contribution differs based on model configurations and the way that this contribution was calculated. Diagnosing the role of a mechanism on regional temperature change using perturbation energy budgets - used in many of these analyses - may lead to a different conclusion than an analysis based on turning off that mechanism in climate models ${ }^{1}$ (Merlis 2014), and we show both in what follows.

Radiation also plays a key role in determining the vertical structure of temperature in the troposphere. In high latitudes, radiative cooling is thought to be critical in determining the mean stratification, particularly inversions (Curry 1983). In low latitudes, the troposphere's stratification is close to moist adiabatic, but biases in the climatologies of comprehensive general circulation models (GCMs) and concomitant differences in projected changes may be related to discrepancies in the treatment of radiative processes between GCMs (Po-Chedley and Fu 2012; O'Gorman and Singh 2013). In addition, changes in cloud radiative effects leave a distinct fingerprint on tropical lapse rate changes in GCM simulations (Mauritsen et al. 2013). Hence radiation is key in determining the vertical structure of the troposphere at high latitudes and has a possible role in determining the tropical stratification. The nonlinearity of the Stefan-Boltzmann law may then impact the vertical structure of temperature change by increasing the

\footnotetext{
${ }^{1}$ This can account for the differing conclusions between Langen et al. (2012) and Pithan and Mauritsen (2014) about the role of the water vapor feedback for polar amplification.
}

temperature response of the cold upper layers of the troposphere relative to the warm lower layers.

Here, the role of the Stefan-Boltzmann law's nonlinearity on the structure of radiatively forced temperature change is assessed. The Stefan-Boltzmann law has been examined as a stabilizing nonlinearity for the global climate sensitivity under large perturbations (Bloch-Johnson et al. 2015), while our focus is on the role of this nonlinearity on the pattern of global warming, rather than its amplitude. First, the spatial variations of the Planck feedback and $\mathrm{CO}_{2}$ 's radiative forcing for Earth's atmosphere are compared using reanalysis data and a radiative kernel derived from a comprehensive GCM, as well as the Planck feedback and radiative forcing derived from a moist idealized GCM (section 2), which reveals a combined effect that would give rise to tropical amplification. We then compare the pattern of warming of an idealized aquaplanet atmospheric GCM (Frierson et al.2006) with a gray radiation scheme to one that has a linearized version of the gray radiation scheme. It is linearized by replacing $E=\sigma T^{4}$ with $E=A+B T$ for the radiation scheme's longwave emission source function (section 3 ). This lets us 1 ) test the importance of the nonlinearity for polar amplification (section 4) and 2) assess its effect on the vertical structure of tropospheric warming (section 5). Finally, we use a radiative-convective and a pure radiative configuration of the idealized model to confirm that the Stefan-Boltzmann nonlinearity affects the vertical structure of tropospheric warming across a hierarchy of model configurations. Radiation plays an increasingly important role in determining the structure of temperature change, as advection and convection are successively deactivated, although all configurations show a "cold-altitudes-warm-more" effect from the $\sigma T^{4}$ nonlinearity.

\section{Patterned warming from the Stefan-Boltzmann law}

A consequence of the nonlinearity of $\sigma T^{4}$ is a less stabilizing Planck feedback at high latitudes, which may cause polar amplification. If changes in the oceanic and atmospheric energy transports and all other radiative feedbacks are ignored, the temperature response to a radiative forcing at the top of atmosphere $\mathcal{F}$ is $\Delta T_{s}=-\mathcal{F} / \lambda_{P}$, where $T_{s}$ is the surface air temperature and $\lambda_{P}$ is the Planck feedback. Hence, if $\mathcal{F}$ is constant over all latitudes, the temperature response is greater at climatologically colder high latitudes than at climatologically warmer low latitudes. This is the mechanism by which the spatial structure of the Planck feedback, through the Stefan-Boltzmann law, has been argued to give rise to PA. 

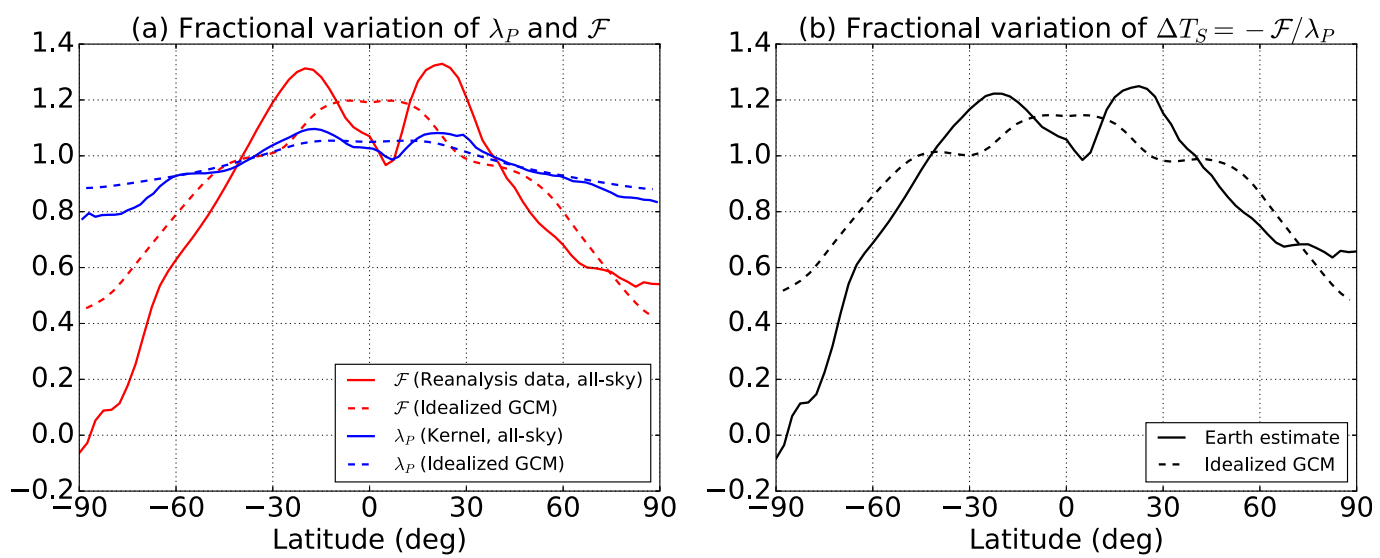

FIG. 1. (a) Fractional variation from the global mean of radiative forcing $\mathcal{F}$ and Planck feedback $\lambda_{P}$ for Earth estimates (solid lines) and the idealized GCM (dashed lines). The $\mathrm{CO}_{2}$ radiative forcing data (solid red) are from Huang et al. (2016) and the Planck feedback (solid blue) is the annual mean of the temperature radiative kernel derived from GFDL's comprehensive AM2 GCM (Soden et al. 2008). The local definition of feedback is used, hence the Planck feedback is equal to the mass-weighted vertical integral of $\left(K_{T} \Delta T / \Delta T_{S}\right)$, where $K_{T}$ is the temperature kernel, $\Delta T=1 \mathrm{~K}$, and $\Delta T_{S}=1 \mathrm{~K}$. (b) Fractional variation of temperature change is given by $\Delta T_{S}=-\mathcal{F} / \lambda_{P}$.

The simple argument for the role of the Planck feedback on polar amplification relies on the assumption that the radiative forcing is latitudinally uniform. However, it is important to note that the TOA longwave radiative forcing $\mathcal{F}$ from an increase in well-mixed greenhouse gases has structure arising from the mean temperature distribution (Zhang and Huang 2014; Payne et al. 2015): the forcing is larger in the tropics than in high latitudes and this counteracts the Planck feedback's tendency for PA. In Payne et al. (2015), a simple one-layer atmospheric model is used to show that the forcing varies with latitude or mean temperature in a way that exactly compensates for the variation in the Planck feedback. In their model, the radiative forcing from a small change in atmospheric emissivity has the same $\left(4 \sigma T^{3}\right)^{-1}$ dependence and, therefore, the same structure as the Planck feedback. This implies that if the Planck feedback is the only active feedback and all energy transports are unchanged, the change in temperature induced by the forcing would be uniform in latitude. Feldl and Roe (2013b) also show this in the partial temperature change induced by forcing and normalized by the Planck feedback (their Fig. 6a). Radiative forcing estimates for carbon dioxide and other well-mixed greenhouse gases have long been known to have spatial structure (e.g., Hansen et al. 2005), although the consequences of this structure on the atmospheric energy transport and circulation changes have only recently been examined (Huang and Zhang 2014; Merlis 2015). Here, we present an analysis of how the spatial structure of radiative forcing affects the meridional structure of the surface air temperature change.
Huang et al. (2016) showed radiative forcing estimates from homogeneous changes in greenhouse gas concentration using a contemporary reanalysis estimate of the atmospheric state and an accurate radiative transfer calculation. They subsequently related the TOA radiative forcing variations to climatological factors beyond the mean temperature and showed that the magnitude of the forcing is strongly dependent on surface and atmosphere variables, particularly the lapse rate. Figure 1a shows the fractional latitudinal variation of instantaneous radiative forcing from doubling $\mathrm{CO}_{2}$ (Huang et al. 2016, their Fig. 1c) and the fractional variation of the Planck feedback from the temperature radiative kernel derived from the Geophysical Fluid Dynamics Laboratory AM2 comprehensive atmospheric GCM (Soden et al. 2008, their Fig. 6) using the local definition of feedback. If the Planck feedback is the only active feedback and all energy transports are unchanged, the surface air temperature change can be calculated as $\Delta T_{s}(\phi)=-\mathcal{F}(\phi) / \lambda_{P}(\phi)$ (Fig. 1b), and has about $40 \%$ less warming in the Arctic than in the tropics. The combination of the Planck feedback and the forcing from a doubling of $\mathrm{CO}_{2}$ would give a tropically amplified surface air warming that arises from the greater equatorto-pole variation of the forcing compared to the Planck feedback (Fig. 1a). The stronger equator-to-pole variation of the forcing arises primarily from the dependence of the forcing on the climatological lapse rate (Huang et al. 2016): the high latitudes have weak forcing because they are closer to the isothermal regime, where there is no greenhouse effect, than low latitudes. In summary, the climatological meridional temperature gradient gives rise to a 
spatially varying Planck feedback that would, in isolation, cause polar amplified warming. However, this is more than offset by the climatological temperature distribution's effect on the radiative forcing, where both the mean temperature and lapse rate's equator-to-pole decrease produce a tropically amplified forcing.

A similar argument to the one made for the role of the Planck feedback on polar amplification can be made for the vertical dependence of temperature change in response to radiative forcing. The effect of the nonlinearity of $\sigma T^{4}$ is that the temperature response of the higher-altitude, colder layers of the troposphere is larger than the temperature response of lower-altitude, warmer layers. Hence, with linearization, the lower-altitude layers would be more sensitive and the higher-altitude layers would be less sensitive to a given forcing. Therefore, one expects eliminating Stefan-Boltzmann law's nonlinearity to result in a destabilization of the tropospheric stratification relative to nonlinear radiation GCM simulations of radiatively forced warming, in the absence of other changes in the atmospheric heat budget. A potentially modulating influence is that the perturbed radiative flux at individual vertical levels may differ in the absence of that nonlinearity. Whereas in the horizontal dimension, one can assume the horizontal energy transport constant and compute the TOA radiative fluxes, the same cannot be said about the vertical dimension because there is radiative coupling between levels, making the separation of different vertical levels difficult. ${ }^{2}$ This provides additional motivation for using a GCM to isolate the effect of the Stefan-Boltzmann nonlinearity. It is also worth mentioning that the perturbation longwave flux from increased longwave optical depth has vertical structure, which could be considered in a more detailed assessment than we perform here.

We aim to test the effect of the nonlinearity of $E=\sigma T^{4}$ on the vertical and horizontal pattern of warming by linearizing this function in an idealized aquaplanet GCM with a gray radiation scheme. The GCM simulations have additional degrees of freedom beyond the purely radiative mechanism by which the $E=\sigma T^{4}$ nonlinearity gives rise to inhomogeneous or patterned warming. In particular, changes in advection and convection can give rise to deviations from the expectations described in this section.

\section{Idealized GCM}

We use the moist idealized GCM described in Frierson et al. (2006) with the modifications and parameter values

\footnotetext{
${ }^{2}$ We thank Karen Shell for pointing this out.
}

described in O'Gorman and Schneider (2008). The surface boundary condition is an aquaplanet with a slab mixed-layer ocean with the heat capacity of $1 \mathrm{~m}$ of water and no representation of ocean heat transport. The GCM's spectral dynamical core has T42 spectral truncation for a nominal horizontal resolution of $2.8^{\circ} \times 2.8^{\circ}$ and 30 vertical levels. The sea surface temperature (SST) is interactively computed using the surface radiative and turbulent fluxes, with the surface turbulent fluxes determined by bulk aerodynamic formulas. A $k$-profile scheme with a dynamically determined boundary layer height is used to parameterize the boundary layer turbulence. The GCM uses a simplified Betts-Miller convection scheme (Frierson 2007). The large-scale condensation is parameterized such that the relative humidity does not exceed one and the condensed water is assumed to immediately return to the surface. Incoming solar radiation is an idealized second Legendre polynomial function that is representative of Earth's annual mean with no seasonal or diurnal cycle. For longwave radiation, the model has gray radiative transfer, with the longwave optical depth as in O'Gorman and Schneider (2008). The radiative fluxes are a function of temperature and pressure alone, so there are no water vapor or cloud feedbacks. The surface has no representation of sea ice and has a uniform surface albedo, so there is no surface albedo feedback. All simulations are 2000 days with time averages over the last 1000 days shown, when all climate states have reached a statistical steady state.

To calculate the value of the radiative forcing, simulations where the SST is prescribed to the time mean and zonal mean of the control simulation SST with both control and perturbed optical depth were performed. The perturbation in radiative flux at the top of the atmosphere after the stratospheric and tropospheric temperature has changed directly in response to the optical depth (as opposed to changes mediated by surface temperature variations) defines the troposphere-adjusted radiative forcing (Hansen et al. 2005), which is also known as the effective radiative forcing. This troposphereadjusted forcing is a more accurate measure of the radiative forcing and alters our estimate of the lapse rate feedback compared to using instantaneous radiative forcing, although the difference in spatial structure between troposphere-adjusted and instantaneous radiative forcing remains small (not shown). This gray radiation model has less stratospheric cooling than comprehensive climate models. However, the focus of this study is the troposphere and any effect this different stratospheric cooling may have on the troposphere is taken into account by using this adjusted forcing. The latitudinal structure of the troposphere-adjusted radiative forcing 

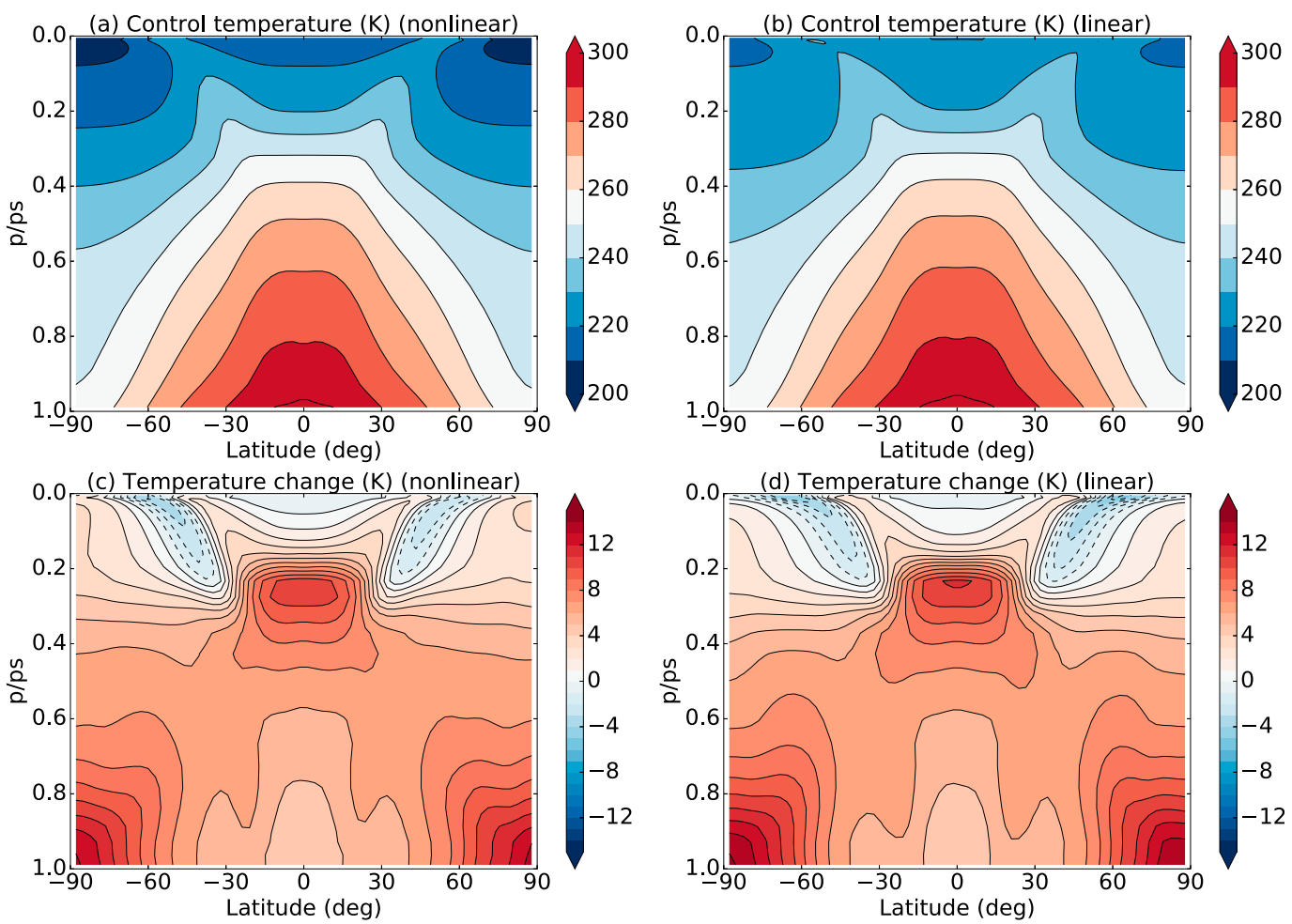

FIG. 2. Time- and zonal-mean temperature of control simulations with (a) nonlinear radiation and (b) linear radiation $\left(B=3.7 \mathrm{~W} \mathrm{~m}^{-2} \mathrm{~K}^{-1}, A=-700 \mathrm{~W} \mathrm{~m}^{-2}\right)$. Also shown is the change in the time- and zonal-mean temperature between the increased and control optical depth simulations with (c) nonlinear radiation and (d) linear radiation $\left(B=3.7 \mathrm{~W} \mathrm{~m}^{-2} \mathrm{~K}^{-1}, A=-700 \mathrm{~W} \mathrm{~m}^{-2}\right)$.

of the idealized GCM closely resembles that of instantaneous $\mathrm{CO}_{2}$ radiative forcing from Huang et al. (2016) (Fig. 1, dashed lines).

Figure 2a shows the time- and zonal-mean temperature field for the control simulation of the standard configuration of the idealized moist GCM. The temperature field is comparable to that of Earth's annual mean, although the tropospheric lapse rate is everywhere positive, even in high latitudes, and the stratosphere is not realistically represented due to the use of gray radiation and the absence of ozone. We can, therefore, assess the role of the Stefan-Boltzmann nonlinearity on the pattern of radiatively forced temperature change for a regime similar to that of Earth's climate.

The radiation source function, $E=\sigma T^{4}$, of longwave radiation is replaced with a linear approximation, $E=A+B T$, for the linear radiation simulations. The temperature dependence of the Planck feedback and, therefore, its spatial structure is suppressed, and we can analyze the effect of this nonlinearity on the vertical structure of warming. Different values for $A$ and $B$ are chosen to test the sensitivity of our result to these constants. The chosen values of $A$ and $B$ are displayed in the legend of Fig. 3. The values of $A$ and $B$ are chosen such that $E=A+B T$ is tangent to $E=\sigma T^{4}$ at $240,250,265$, and $300 \mathrm{~K}$ to span most of the control simulation's temperature field (Fig. 2a), and these values are then rounded for ease of replication. We also test the sensitivity to changes in $A$ with the same value of $B$ with two more linearizations. With $B=4.6 \mathrm{~W} \mathrm{~m}^{-2} \mathrm{~K}^{-1}$, the simulated temperature change pattern resembles the nonlinear radiation simulation most closely, so we test the sensitivity of the results to variations in $A$ for this value of $B$. In total, we present six linearizations. We note that none of the simulations reach a low enough temperature to have a negative value for longwave emission $(A+B T>0$ for all $T)$. While alternative linear radiation simulations with $E=\sigma T^{4}$ linearized about the spatially varying climatological temperature are possible, it is important that $B$ is constant with latitude as the Planck feedback is then spatially uniform and thus has no effect on the horizontal structure of temperature change.

Figures $2 \mathrm{a}$ and $2 \mathrm{~b}$ show the control temperature field for the nonlinear radiation simulation and a linear radiation simulation $\left(A=-700 \mathrm{~W} \mathrm{~m}^{-2}, B=3.7 \mathrm{~W} \mathrm{~m}^{-2} \mathrm{~K}^{-1}\right)$, respectively. These control temperature fields are broadly 

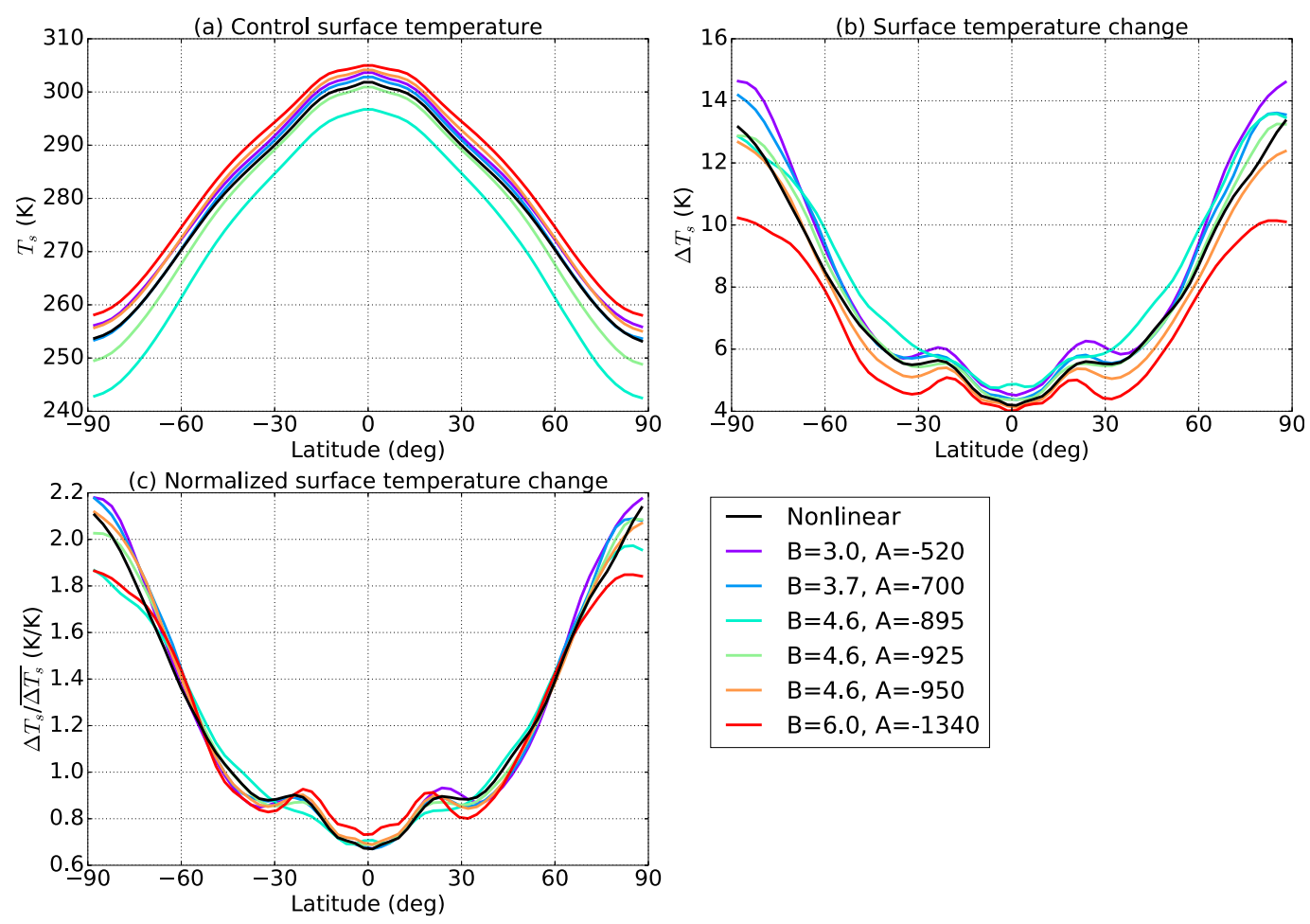

FIG. 3. (a) Surface air temperature for the control simulation with different linearizations of the longwave emission source function. (b) Surface air temperature change between the increased and control optical depth simulations. (c) Surface air temperature change normalized by the global-mean surface air temperature change.

similar, although small changes occur due to biases in the longwave radiation emission function as a result of its linearization. The control temperature fields of the linear radiation simulations are comparable, so one of them is chosen here as an example. Figure $3 \mathrm{a}$ also shows that the control surface air temperature is sensitive to the $A$ parameter (note the spread between the three simulations with $B=4.6 \mathrm{~W} \mathrm{~m}^{-2} \mathrm{~K}^{-1}$ ). The $A$ parameter sets the amount of temperatureindependent emission of radiation, so a higher $A$ means greater longwave emission and a colder control climate. The $B$ parameter sets the amount of temperature change required for a certain change in emission and has a significant impact on the amount of global warming (Fig. 3b).

The radiative forcing due to an increase in greenhouse gas concentration is modeled by increasing the longwave optical depth. In this model, the optical depth at the surface varies in latitude as $\tau_{\text {eq }}+\left(\tau_{\text {pole }}-\tau_{\text {eq }}\right) \sin ^{2}(\phi)$, where $\tau_{\text {eq }}$ and $\tau_{\text {pole }}$ are the optical depth at the equator and pole respectively and $\phi$ is the latitude. The control values for $\tau_{\text {eq }}$ and $\tau_{\text {pole }}$ are 7.2 and 1.8 respectively. The vertical structure of the optical depth has a component that decays quartically in pressure as $\left(p / p_{s}\right)^{4}$, mimicking water vapor's scale height, and a component that decreases linearly in pressure as $p / p_{s}$, mimicking wellmixed greenhouse gases, where $p$ is pressure and $p_{s}$ is the surface pressure [Eq. (1) of O'Gorman and Schneider (2008)]. The effect of an increase in greenhouse gas concentrations is represented by a multiplicative increase of the optical depth by a factor of 1.4 for both $\tau_{\mathrm{eq}}$ and $\tau_{\text {pole. }}$. This results in a global-mean troposphere-adjusted radiative forcing of $24 \mathrm{~W} \mathrm{~m}^{-2}$ for the nonlinear radiation simulation and from 20 to $27 \mathrm{~W} \mathrm{~m}^{-2}$ for the linear radiation simulations with some dependence on the linearization coefficients (section 5). The main results of this paper are insensitive to the magnitude of the forcing: we have repeated all analyses in perturbation simulations where the optical depth is multiplied by 1.1, which leads to a global-mean forcing of $5.9 \mathrm{~W} \mathrm{~m}^{-2}$, which is similar to a quadrupling of $\mathrm{CO}_{2}$ (see appendix). While the global-mean forcing is large in the perturbation simulations presented in this paper, the equator-topole variation in forcing is similar to estimates for Earth (Fig. 1a), although the forcing in the Southern Hemisphere is higher in the Earth estimates, which is partially due to the high altitude of the Antarctic continent and stratospheric temperature structure (Smith et al. 2018). It is also worth noting that we compare the forcing from a model with gray radiation, which has no cloud 
representation, to an all-sky Earth estimate of $\mathrm{CO}_{2}$ forcing.

Figures $2 \mathrm{c}$ and $2 \mathrm{~d}$ show the temperature change field for the nonlinear radiation simulations and corresponding linear radiation simulations. Both temperature change patterns are broadly similar. Despite the idealized nature of the GCM, they are in good agreement with the ensemble-mean pattern of temperature change in the comprehensive GCMs shown in the Fifth Assessment Report of the Intergovernmental Panel on Climate Change (IPCC) (IPCC 2013, their Fig. 12.12); however, Figs. $2 \mathrm{c}$ and $2 \mathrm{~d}$ represent temperature change between two equilibrium states while the IPCC figures represent the transient climate response, so the surface air temperature change in the Southern Hemisphere is not polar amplified in the IPCC figures. The idealized GCM has cooling in part of the stratosphere, although its structure and magnitude are unrealistic due to the simplicity of this model's radiative transfer scheme.

Figure $3 \mathrm{~b}$ shows the surface air temperature change between the control and increased longwave optical depth simulations for nonlinear radiation (black) and the different linearizations of $\sigma T^{4}$ (colors). The simulated polar warming varies from 11 to $15 \mathrm{~K}$ in these simulations. However, much of this results from variation in the amount of global warming rather than from variation in the pattern of warming. Figure $3 \mathrm{c}$ shows the surface air temperature change normalized by its global mean. For all linearizations, the simulated pattern of warming is quite similar to the nonlinear radiation simulation. These experiments then show that the linearization of the radiation scheme does not substantially affect the pattern of surface air warming. The amount of normalized surface temperature change at the poles varies by approximately $15 \%$ across the radiation parameters $\left(0.3 \mathrm{KK}^{-1}\right.$ relative to a total normalized polar warming of $2 \mathrm{KK}^{-1}$ ). However, the range of linear radiation simulations includes both those with slightly more and those with less polar amplified warming. This result therefore contradicts the expectation that the nonlinearity of $E=\sigma T^{4}$ systematically increases the amount of polar amplification of surface air warming.

\section{Horizontal structure of warming}

We perform a radiative feedback analysis to diagnose both the factors affecting the climate sensitivity and the spatial structures of the different feedbacks. The GCM used in this experiment has gray radiation and a prescribed optical depth, so only the temperature feedback is active, and there are no water vapor, cloud, or surface albedo feedbacks. The temperature feedback consists of the Planck feedback parameter $\lambda_{P}$ and the lapse rate feedback parameter $\lambda_{\mathrm{LR}}$. The total feedback parameter $\lambda_{T}=\lambda_{P}+\lambda_{\mathrm{LR}}$ is computed as follows:

$$
\lambda_{T}(\phi)=\frac{-\mathcal{F}(\phi)+\Delta\left[\nabla \cdot \mathbf{F}_{\mathrm{MSE}}(\phi)\right]}{\Delta T_{s}(\phi)},
$$

where $\mathcal{F}$ is the radiative forcing, $\Delta\left[\nabla \cdot \mathbf{F}_{\mathrm{MSE}}\right]$ is the change in the divergence of the vertically integrated moist static energy (MSE) flux between the control and increased optical depth simulations, which is the GCM's horizontal energy transport. The change in the TOA net radiation is used to calculate the change in divergence of the MSE flux, although an explicit calculation of the MSE flux convergence using the instantaneous GCM simulated winds and MSE fields has very similar results (not shown). This is a local feedback analysis where the sum of the forcing and the change in MSE flux divergence is balanced by the product of the local change in surface temperature and the locally defined climate feedback parameter. The feedback used here is described as "local" in Feldl and Roe (2013a), in which other feedback analysis methods are described.

To compute the Planck feedback, a second temperature field is initialized at each time step in the GCM's radiation scheme with a temperature value incremented by $1 \mathrm{~K}$ at the surface and for all of the GCM's vertical levels. The Planck feedback is then computed as the time mean of the difference between the outgoing longwave radiation (OLR) corresponding to the GCM's prognostic temperature field in the control simulation and the OLR corresponding to the temperature field incremented by one. For the linear radiation simulations, the Planck feedback is trivially equal to the $B$ parameter of the $E=\sigma T^{4}$ linearizations, given that we use a local feedback analysis. The lapse rate feedback is computed as the residual: $\lambda_{\mathrm{LR}}=\lambda_{T}-\lambda_{P}$. Therefore, nonlinearities that occur due to individual processes (e.g., the Stefan-Boltzmann law) and nonlinear interactions among different processes are not separated out (Feldl and Roe 2013b). However, the feedbacks are very similar in simulations with smaller forcing (see appendix), suggesting that the method used to diagnose the feedback is accurate.

We diagnostically employ the forcing and feedback analysis of the GCM results by expressing each component's change in the local TOA radiation budget in terms of a surface temperature change. The Planck feedback can be decomposed into its global mean [indicated by $\overline{(\cdot)}$ and calculated as the latitudinal average of the locally defined Planck feedback $\left.\lambda_{P}(\phi)\right]$ and its deviation from this mean $\lambda_{P}-\overline{\lambda_{P}}$. Similar to the analysis of Feldl and Roe (2013b), we diagnose the change in surface air warming by isolating $\overline{\lambda_{P}} \Delta T_{s}$ in Eq. (1) and dividing by $\overline{\lambda_{P}}$ as follows: 
(a) Decomposition of warming

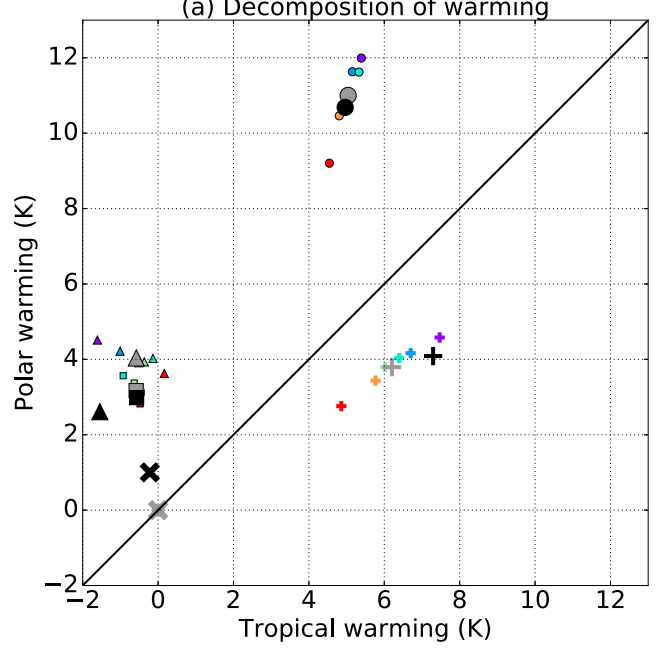

(b) Normalized decomposition of warming

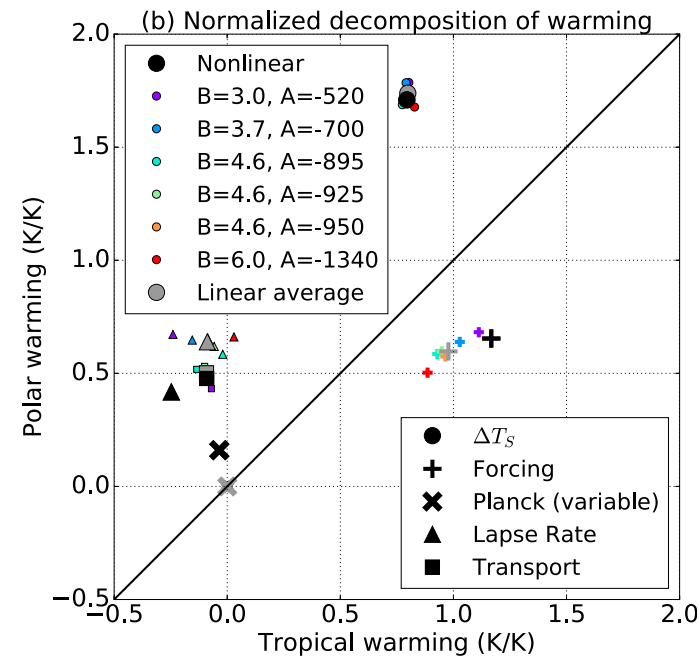

FIG. 4. (a) Energy budget diagnosis of polar $\left(|\phi|>60^{\circ}\right)$ and tropical warming $\left(|\phi|<30^{\circ}\right)$ with changes from spatially varying forcing, the variable Planck feedback, the lapse rate feedback, and the convergence of moist static energy transport [Eq. (2)]. (b) Energy budget diagnosis normalized by global-mean surface air temperature change. The distance from the 1:1 line (black) indicates the component's diagnosed contribution to the polar or tropical amplification of warming. The gray symbols are an average of the linear radiation simulations.

$$
\Delta T_{s}(\phi)=\frac{-\mathcal{F}(\phi)+\Delta\left[\nabla \cdot \mathbf{F}_{\mathrm{MSE}}(\phi)\right]-\Delta T_{s}(\phi)\left\{\lambda_{\mathrm{LR}}(\phi)+\left[\lambda_{P}(\phi)-\overline{\lambda_{P}}\right]\right\}}{\overline{\lambda_{P}}} .
$$

From the left to the right, the four terms in the numerator will be denoted as forcing, transport, lapse rate feedback, and variable Planck feedback, respectively. This type of budget analysis also formed the basis of the quantification of Arctic warming in Pithan and Mauritsen (2014). We note that this analysis can give a different quantitative interpretation for the combination of the Planck feedback and the forcing than the purely radiative estimate given by the ratio $-\mathcal{F}(\phi) / \lambda_{P}(\phi)$ (section 2). The difference can arise because the Planck feedback's deviation from its mean in Eq. (2) depends on the latitudinal structure of temperature change, which in turn is affected by other processes like changes in atmospheric energy transport.

Figure 4a shows the area-weighted polar mean $\left(|\phi|>60^{\circ}\right)$ versus the area-weighted tropical mean $\left(|\phi|<30^{\circ}\right)$ of each term of this sum. Tests with different averaging conventions show that results in Fig. 4 are insensitive to the latitude ranges. The distance to the $1: 1$ line indicates the forcing or feedback's contribution to polar amplification (Pithan and Mauritsen 2014). The black symbols correspond to the nonlinear radiation simulation and other colors correspond to different linear radiation simulations. The gray symbols are an average of the linear radiation simulations contributions, and this average is insensitive to the details of which linear radiation simulations are used to form it, as long as the chosen simulations span the range of $B$ values. We note that if the forcing is increased in a model that has an amplitude-independent ratio of polar to tropical warming, the points corresponding to the simulation with increased forcing will be further from the 1 : 1 line even though the pattern of warming is the same. Likewise, two models with identical warming patterns, but different global-mean climate sensitivity would appear to have different degrees of polar amplification on this diagram. Therefore, we normalize all points by the global-mean surface air temperature change in Fig. $4 \mathrm{~b}$ and discuss this in what follows:

- All linear radiation simulations have a zero variable Planck feedback contribution by definition $\left(\lambda_{P}-\overline{\lambda_{P}}=0\right)$. The nonlinear radiation simulations' variable Planck contribution is above the 1:1 line and contributes to polar amplification according to this energy budget analysis (black $\mathrm{x}$ ). We note that the variable Planck feedback term depends on the latitudinal profile of the Planck feedback $\lambda_{P}(\phi)$ and temperature change $\Delta T_{s}(\phi)$ [Eq. (2)].

- The lapse rate-related temperature change is more positive in linear radiation simulations than in nonlinear radiation simulations over all latitudes (colored triangles are farther up and to the right compared to the black triangle, except for the extreme $B=3.0 \mathrm{~W} \mathrm{~m}^{-2} \mathrm{~K}^{-1}$ 

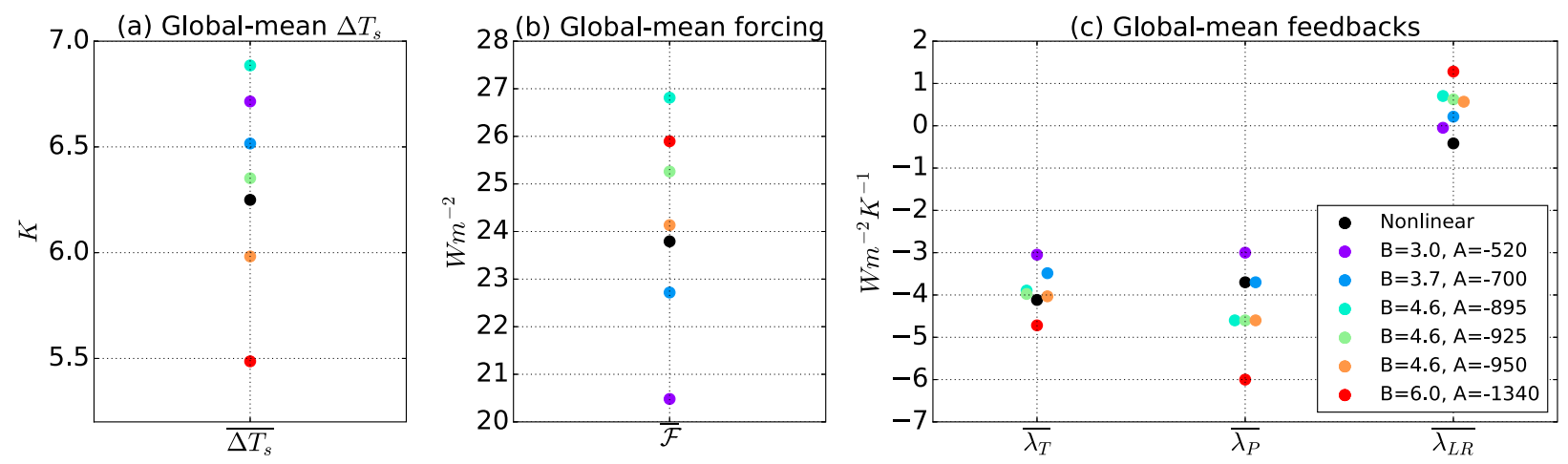

FIG. 5. (a) Global-mean surface air temperature change $\overline{\Delta T_{s}}$, (b) global-mean radiative forcing $\overline{\mathcal{F}}$, and (c) global-mean radiative feedbacks $\left(\overline{\lambda_{T}}, \overline{\lambda_{P}}, \overline{\lambda_{\mathrm{LR}}}\right)$ for all simulations, where feedbacks are computed using the global definition of feedback.

case) due to the colder upper tropospheric layers having a larger magnitude temperature increase in the nonlinear radiation case (section 5).

- Once normalized, the change in moist static energy transport is similar between linear radiation and nonlinear radiation simulations (except for the extreme $B=3.0 \mathrm{~W} \mathrm{~m}^{-2} \mathrm{~K}^{-1}$ case). This is consistent with diffusive closures-the change in moist static energy transport is proportional to the pattern of temperature change (Fig. 3), all else being equal.

- The forcing changes slightly between the different simulations - the effect of increasing the optical depth varies depending on the radiation scheme as a result of slight differences in the control simulation temperature field. However, the variation in the forcing term in Fig. 4 arises primarily from the generally higher value of $\overline{\lambda_{P}}$ for the linearizations presented here, as evidenced by the fact that the spread of the linear radiation simulations' terms form a line that intersects the origin. Therefore, the forcing term $-\mathcal{F}(\phi) / \overline{\lambda_{P}}$ is generally smaller (closer to the origin) in the linear radiation simulations, as can be seen in Figs. $4 \mathrm{a}$ and $4 \mathrm{~b}$. The spread of the linear radiation simulations' transport term also forms a line that intersects the origin in Fig. 4a, but these collapse together when normalized by the global-mean surface air temperature change (Fig. 4b), which suggests that the variation of $\overline{\lambda_{P}}$ does not affect the normalized transport term. The lapse rate feedback terms form a line that does not intersect with the origin, which suggests this spread does not primarily arise from the variation in $\overline{\lambda_{P}}$.

In summary, the Planck feedback functions as expected: it promotes polar amplification in the nonlinear radiation simulations and has no effect on polar amplification in the linear radiation simulations. According to the budget analysis, this difference could be offset by the change in MSE flux convergence, radiative forcing and/or lapse rate feedback to arrive at a comparable warming pattern (Fig. 3c). Figure $4 \mathrm{~b}$ shows there are only modest differences in the MSE flux convergence change between the linear and nonlinear radiation simulations. The radiative forcing in the linear radiation simulations contributes to slightly less tropical warming than in the nonlinear radiation simulation, but not enough to compensate for the Planck feedback. The only other degree of freedom in this GCM is the lapse rate feedback. The difference in the variable Planck feedback contribution between the nonlinear and linear radiation simulations is offset by changes in the lapse rate feedback: the tendency of the lapse rate feedback in the linear radiation simulations is generally toward more polar amplification than in the nonlinear radiation simulation. The structure of the lapse rate feedback is discussed in more detail in the next section.

\section{Global-mean feedbacks and vertical structure of warming}

Figure 5 shows the forcing-feedback analysis for the global-mean values of the temperature change $\overline{\Delta T_{s}}$, radiative forcing $\overline{\mathcal{F}}$, and radiative feedbacks $\left(\overline{\lambda_{T}}, \overline{\lambda_{P}}, \overline{\lambda_{\mathrm{LR}}}\right)$ for all simulations. Here, we use the global definition of feedback to compute the total feedback:

$$
\overline{\lambda_{T}}=\frac{\overline{-\mathcal{F}(\phi)+\Delta\left[\nabla \cdot \mathbf{F}_{\mathrm{MSE}}(\phi)\right]}}{\overline{\Delta T_{s}(\phi)}} .
$$

The perturbed simulations have about $6 \mathrm{~K}$ of global warming that results from a combination of a large globalmean radiative forcing $\left(\approx 24 \mathrm{~W} \mathrm{~m}^{-2}\right)$ and a very stabilizing climate feedback parameter $\left(\approx-4 \mathrm{~W} \mathrm{~m}^{-2} \mathrm{~K}^{-1}\right)$. A notable feature of Fig. 5 is that the nonlinear radiation simulation (black) is within the range of values of the linear radiation simulations (color) for all variables except the lapse rate feedback $\overline{\lambda_{\mathrm{LR}}}$. The lapse rate feedback of the nonlinear radiation simulation is more negative than that of the linear 

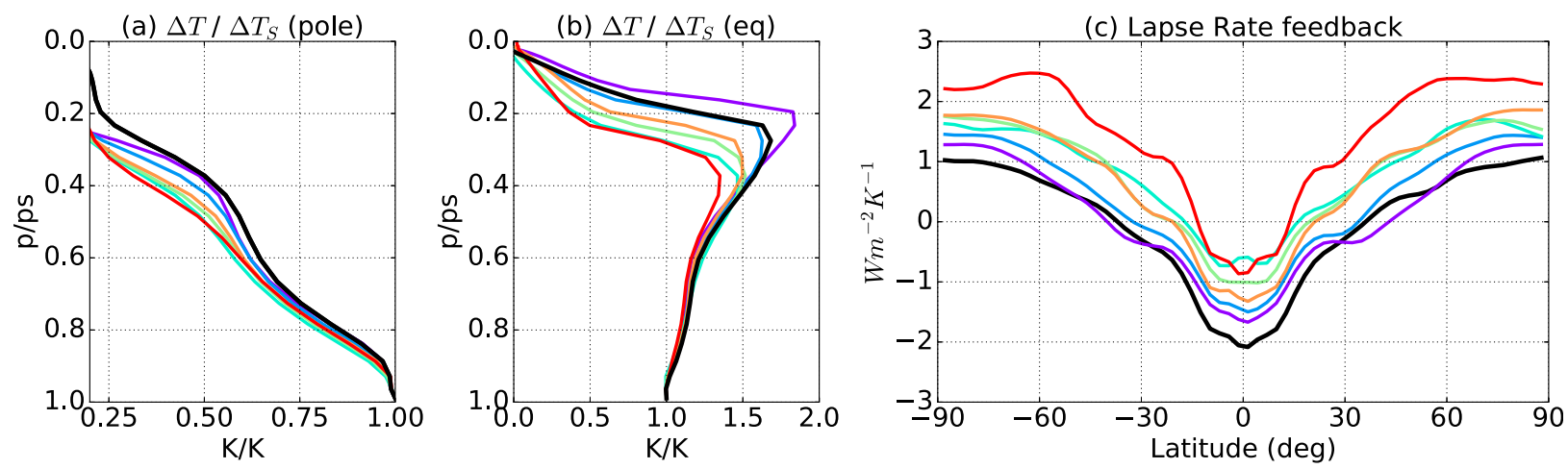

FIG. 6. (a) Area-weighted polar mean $\left(|\phi|>60^{\circ}\right)$ and (b) the area-weighted tropical mean $\left(|\phi|<30^{\circ}\right)$ of the temperature change normalized by the surface air temperature change for the nonlinear radiation simulation (black) and the linear radiation simulations (color). (c) Lapse rate feedback vs latitude for all simulations. Color legend is the same as in Fig. 3.

radiation simulations (apart from the extreme $B=$ $3.0 \mathrm{~W} \mathrm{~m}^{-2} \mathrm{~K}^{-1}$ simulation where the difference is minimal). The importance of the Stefan-Boltzmann nonlinearity on the vertical structure of warming and concomitant effect on $\lambda_{\mathrm{LR}}$ has not been previously documented and is a task that we pursue here.

Figures $6 \mathrm{a}$ and $6 \mathrm{~b}$ show the area-weighted polar mean $\left(|\phi|>60^{\circ}\right)$ and the area-weighted tropical mean $\left(|\phi|<30^{\circ}\right)$ of the temperature change normalized by the surface air temperature change for the nonlinear radiation simulation (black) and the linear radiation simulations (colors). The nonlinear radiation simulation shows a smaller lapse rate increase near the poles and a larger lapse rate decrease in the tropical troposphere (except in the upper troposphere for the extreme $B=3.0 \mathrm{~W} \mathrm{~m}^{-2} \mathrm{~K}^{-1}$ case). Figure $6 \mathrm{c}$ confirms this by showing that the lapse rate feedback of the linear radiation simulations are generally more positive than the nonlinear radiation simulation across all latitudes, aside from the simulation with $B=3.0 \mathrm{~W} \mathrm{~m}^{-2} \mathrm{~K}^{-1}$. The values for the nonlinear radiation simulation can be compared to the CMIP5 multimodel mean (Feldl and Bordoni 2016, their Fig. 1). The nonlinear radiation simulation has a value at the equator close to $-2 \mathrm{~W} \mathrm{~m}^{-2} \mathrm{~K}^{-1}$ and a value at the pole of $1 \mathrm{~W} \mathrm{~m}^{-2} \mathrm{~K}^{-1}$, a somewhat larger equator-topole variation than the multimodel mean, which varies from -1.5 to $0.8 \mathrm{~W} \mathrm{~m}^{-2} \mathrm{~K}^{-1}$ (in the Northern Hemisphere). The transition from negative to positive lapse rate feedback occurs closer to the equator in all of the idealized GCM simulations, which helps account for the less stabilizing global-mean lapse rate feedback relative to comprehensive GCMs.

The interpretation that the difference in lapse rate arises from the cold-altitudes-warm-more mechanism (described in section 2) is supported by two lines of evidence. First, the changes in longwave radiative flux at different levels are approximately the same for the linear and nonlinear radiation simulations, as shown in Fig. 7. Figure 7a shows the change in net longwave radiative flux between the control and increased optical depth experiments for the nonlinear radiation configuration. Figure $7 \mathrm{~b}$ shows the mean of the differences between the change in longwave radiative flux in the nonlinear radiation simulations and the linear radiation simulations. This mean is insensitive to the details of
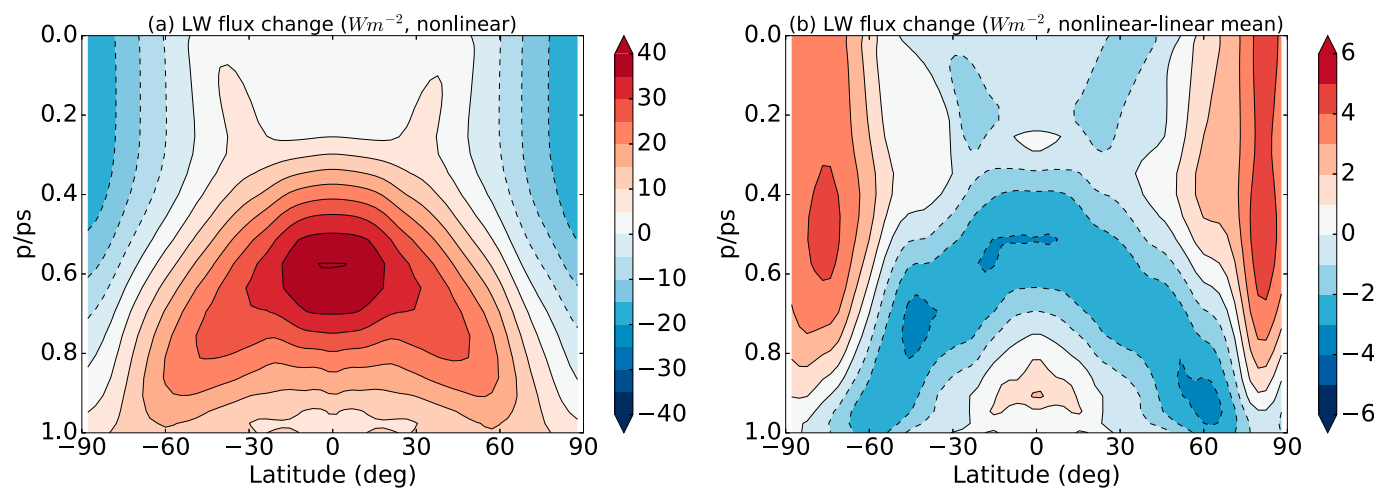

FIG. 7. (a) Change in net longwave (LW) radiative flux (positive upward) between control and increased optical depth experiments for the nonlinear radiation simulations and (b) ensemble mean of the differences between nonlinear and linear radiation simulations in the change in net LW radiative flux. 
which linear radiation simulations are used to form it, as long as the chosen simulations span the range of $B$ values. In the tropics, the difference in the change in longwave radiative flux decreases with altitude, which induces a more bottom-heavy warming in the nonlinear radiation simulation and is opposite to how the $E=\sigma T^{4}$ nonlinearity affects the vertical structure of warming. In the poles, however, the difference in the change in longwave radiative flux increases with altitude, which contributes to the more top-heavy warming in the nonlinear radiation simulation by approximately the same amount as the $E=\sigma T^{4}$ nonlinearity. Hence differences in radiative flux changes do not primarily cause the differences in temperature change in the tropics, but play a comparable role near the poles.

Second, we can eliminate potentially offsetting local vertical tendencies from advection and convection by using different model configurations. To better understand the impact of linearizing $E=\sigma T^{4}$ on the lapse rate feedback, a radiative-convective equilibrium (RCE) configuration and a pure radiative equilibrium $(\operatorname{Rad} \mathrm{Eq})$ configuration of the GCM are analyzed. Figure 8 shows the lapse rate feedbacks for the three different configurations of the GCM in the nonlinear radiation case and a linear $\left(B=3.7 \mathrm{~W} \mathrm{~m}^{-2} \mathrm{~K}^{-1}\right.$, $A=-700 \mathrm{~W} \mathrm{~m}^{-2}$ ) radiation case, chosen because it has a lapse rate feedback close to the nonlinear radiation case. The forcing and feedback in the Rad Eq and RCE configurations are computed using the same methodology as for the full GCM. Since the Rad Eq and RCE configurations are effectively column models, we choose to arrange the columns to have the same control surface air temperature as the full GCM configuration, omitting the warmest and coldest Rad Eq and RCE columns. This is nearly equivalent to altering the insolation in the column models to make their surface air temperature match the control GCM simulation, although the optical depth at a given latitude also differs between the column models and the GCM.

In the Rad Eq configuration of the GCM, the convection and advection processes are deactivated. The vertical temperature profile is thus determined only by how the longwave radiation of each column balances the absorbed solar radiation. The vertical structure of temperature change in the radiative equilibrium configuration decreases by about a factor of 2 from the surface to the $p / p_{s}=0.5$ level (see also Weaver and Ramanathan 1995). In this configuration, the lapse rate feedback is destabilizing over all latitudes, and the nonlinear radiation simulation has a less destabilizing feedback as the colder upper layers of the atmosphere need a larger

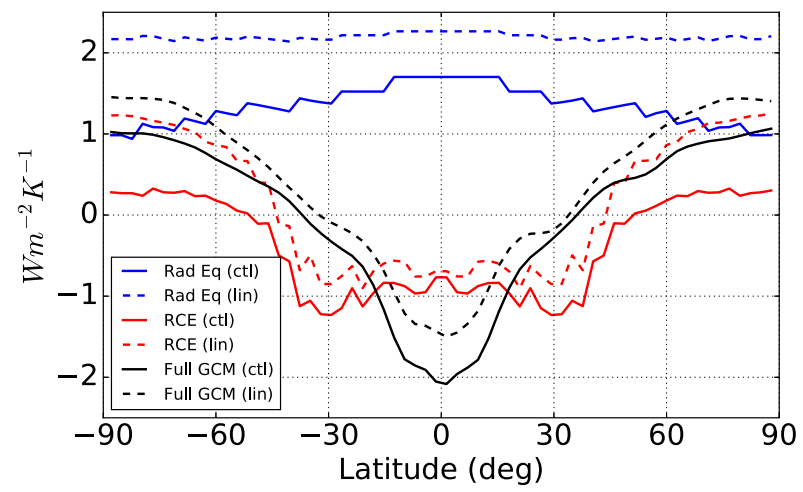

FIG. 8. Lapse rate feedback for three model configurations: Rad Eq, RCE, and the full GCM. For each configuration, the nonlinear radiation simulations (ctl) and linear radiation simulations (lin; with $B=3.7 \mathrm{~W} \mathrm{~m}^{-2} \mathrm{~K}^{-1}, A=-700 \mathrm{~W} \mathrm{~m}^{-2}$ ) are shown.

magnitude increase in temperature to reach the same increase in emission of longwave radiation.

The RCE configuration follows the method described in O'Gorman and Schneider (2008). Convection lessens the difference in lapse rate feedback between the linear and nonlinear radiation cases in the tropics to approximately $0.2 \mathrm{~W} \mathrm{~m}^{-2} \mathrm{~K}^{-1}$. However, a difference of about $1 \mathrm{~W} \mathrm{~m}^{-2} \mathrm{~K}^{-1}$ remains in the polar regions. The difference in lapse rate feedback between the linear and nonlinear radiation simulations in the full GCM configuration is reduced near the poles to approximately $0.3 \mathrm{~W} \mathrm{~m}^{-2} \mathrm{~K}^{-1}$ and is slightly increased in the tropics compared to the RCE configuration. For the linear and nonlinear radiation cases, the lapse rate feedback is less stabilizing in the full GCM configuration than in the RCE configuration outside of the tropics.

We note that the control temperature in our simulations does not have a high-latitude inversion, unlike the Earth's climatology. Having a high-latitude inversion would make the climatological vertical structure of temperature more homogeneous and this would reduce the high-latitude lapse rate feedback's sensitivity to linearizing radiation because the temperature at the emission level would be more similar to the surface.

Presenting the lapse rate feedbacks across this range of atmospheric model configurations shows that the initial difference in lapse rate feedback between the linear and nonlinear radiation simulations in the radiative equilibrium configuration, where cold altitudes warm more as a consequence of the $E=\sigma T^{4}$ nonlinearity (section 2), propagates to the full GCM. This difference is, however, attenuated by the other processes involved in determining the vertical structure of temperature change: convection and advection. Furthermore, this model hierarchy clearly shows the crucial role convection plays in determining the sign of the lapse 
rate feedback by increasing the warming in the upper troposphere in the tropics. The GCM's lapse rate feedback in polar regions, in contrast, is closer to that of the pure radiative equilibrium, suggesting that radiation is a key factor in determining the changes in the vertical stratification of temperature at high latitudes.

\section{Conclusions}

Isolating the factors governing the inhomogeneous pattern of atmospheric temperature change is a central problem in climate dynamics, with implications for the atmospheric general circulation and regional climate changes. Here, we assess the role of the nonlinearity of the Stefan-Boltzmann law $E=\sigma T^{4}$ in determining the pattern of radiatively forced warming. It had previously been suggested to augment polar amplification by giving rise to the spatial structure of the Planck feedback. If all other radiative feedbacks are neglected and energy transports do not change, the nonlinearity of $E=\sigma T^{4}$ implies that the temperature response to a given forcing is higher for a lower mean temperature and lower for a higher mean temperature. The poles are colder than the equator, so this is a reason to expect a greater temperature response at higher latitudes, as recent energy budget analyses of GCMs have found. However, the climatological atmospheric temperature also underlies the equator-to-pole radiative forcing contrast, which is sufficient to produce tropically amplified warming if atmospheric and oceanic energy transports do not change and only the spatially varying Planck feedback and forcing are considered (Fig. 1).

We further examine the role of the nonlinearity of $\sigma T^{4}$ by linearizing the temperature dependence of the blackbody emission of radiation, $E=\sigma T^{4}$, in an idealized aquaplanet GCM with a gray radiation scheme. This mechanism denial experiment shows that the pattern of surface air temperature change does not change when the Stefan-Boltzmann law is linearized. Other components of the changing local energy budget such as a change in the moist static energy flux convergence, the lapse rate feedback or the radiative forcing can offset the Planck feedback's contribution to polar amplification. In these GCM simulations, the lapse rate feedback in the linear radiation simulations contributes to more polar amplification, which offsets the expected decrease in polar amplification from linearizing $E=\sigma T^{4}$.

Finally, we show that the nonlinearity of the StefanBoltzmann law affects the vertical structure of atmospheric temperature change in a manner that tends to make the lapse rate feedback more stabilizing across all latitudes. This suggests that the Stefan-Boltzmann nonlinearity is responsible for increasing the temperature response of the colder upper troposphere and reducing the temperature response of the warmer lower troposphere. We confirm this by examining the lapse rate feedbacks of pure radiative and radiativeconvective configurations of the GCM. The difference in the lapse rate feedbacks between the linear and nonlinear radiation simulations in the pure radiative configuration is well understood: the cold upper layers of the troposphere warm more in the nonlinear radiation simulations to reach a similar perturbation longwave flux. This difference then propagates to the full GCM configuration, although it is attenuated by advection and convection. The sensitivity of the magnitude of the tropical upper-tropospheric warming to the treatment of radiative transfer in these idealized GCM simulations is noteworthy and suggests further examination in radiation's role on the tropical stratification.

Acknowledgments. This work was supported by Fonds de Recherche du Québec-Nature et Technologies (FRQNT) Nouveau Chercheur award and a Natural Sciences and Research Council (NSERC) Discovery grant, as well as a Compute Canada allocation. We thank Yi Huang for providing the $\mathrm{CO}_{2}$ radiative forcing, Brian Soden for providing the AM2 radiative kernel, Paul O'Gorman for providing the RCE code, and Kyle Armour and Tim Cronin for helpful discussions. We thank an anonymous reviewer for encouraging the use of adjusted radiative forcing. We also thank two anonymous reviewers and Karen Shell for constructive reviews.

\section{APPENDIX}

\section{Dependence of Feedback Analysis on Forcing Amplitude}

In Fig. A1, we show the lapse rate feedbacks from all simulations as in Fig. 6c (solid lines), along with lapse rate feedbacks computed with smaller forcing (dashed lines), where perturbed simulations have $1.1 \mathrm{x}$ control optical depth instead of $1.4 \mathrm{x}$ control optical depth. The simulations with smaller forcing have noisier lapse rate feedbacks, but they are not systematically different than simulations with larger forcing. The noisiness of the results with smaller forcing is the reason we chose to present the simulations with larger forcing.

Our feedback calculation technique assesses the $\lambda_{\mathrm{LR}}$ as a residual $\left(\lambda_{\mathrm{LR}}=\lambda_{T}-\lambda_{P}\right.$, where $\lambda_{T}$ is the total feedback and $\lambda_{P}$ is the Planck feedback). Therefore, it includes terms that are second order in perturbation temperature (i.e., nonlinear terms). The smaller forcing 


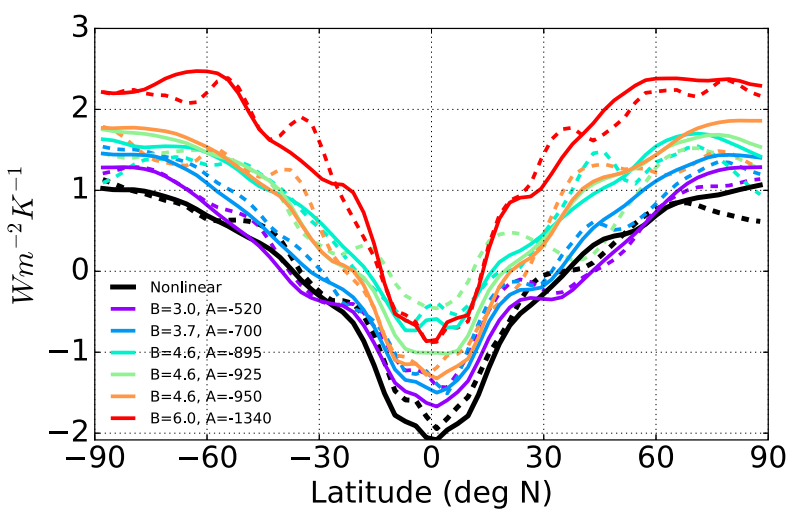

FIG. A1. Lapse rate feedback vs latitude for all simulations as in Fig. 6c (solid lines) and lapse rate feedbacks for simulations with smaller forcing (perturbed simulations with $1.1 \mathrm{x}$ control optical depth instead of $1.4 x$ control optical depth, dashed lines).

simulations have warming that is about a quarter of the larger forcing simulations, so the nonlinear term should be less than $10 \%$ of the magnitude (Roe and Baker 2007). That the smaller forcing simulations have similar lapse rate feedback implies these nonlinear terms have sufficiently low amplitude in these simulations. Hence, our linear feedback analysis is appropriate for this gray radiation model and large forcing value.

We have also computed the Planck feedback with $\Delta T=6 \mathrm{~K}$, which is comparable to the global-mean surface air temperature change in the simulations presented in the main text. The Planck feedback has the same pattern but is slightly more stabilizing (more negative feedback value) by $2 \%-3 \%$. This difference is due to the second-order expansion of $E=\sigma T^{4}$. Therefore, we do not use a larger temperature change to assess the Planck feedback because it implicitly includes nonlinear terms - at odds with the standard definition of the Planck feedback as the first-order, linear response of net radiation to a temperature change.

\section{REFERENCES}

Alexeev, V. A., and C. H. Jackson, 2013: Polar amplification: Is atmospheric heat transport important? Climate Dyn., 41, 533547, https://doi.org/10.1007/s00382-012-1601-z.

Bloch-Johnson, J., R. T. Pierrehumbert, and D. S. Abbot, 2015: Feedback temperature dependence determines the risk of high warming. Geophys. Res. Lett., 42, 4973-4980, https:// doi.org/10.1002/2015GL064240.

Curry, J., 1983: On the formation of continental polar air. J. Atmos. Sci., 40, 2278-2292, https://doi.org/10.1175/1520-0469(1983)040<2278: OTFOCP $>2.0 . \mathrm{CO} ; 2$.

Feldl, N., and G. H. Roe, 2013a: Four perspectives on climate feedbacks. Geophys. Res. Lett., 40, 4007-4011, https://doi.org/ 10.1002/grl.50711.

$\longrightarrow$, and $-2013 \mathrm{~b}$ : The nonlinear and nonlocal nature of climate feedbacks. J. Climate, 26, 8289-8304, https://doi.org/ 10.1175/JCLI-D-12-00631.1.
— , and S. Bordoni, 2016: Characterizing the Hadley circulation response through regional climate feedbacks. J. Climate, 29, 613-622, https://doi.org/10.1175/JCLI-D-15-0424.1.

Frierson, D. M., 2007: The dynamics of idealized convection schemes and their effect on the zonally averaged tropical circulation. J. Atmos. Sci., 64, 1959-1976, https://doi.org/10.1175/ JAS3935.1.

— I. M. Held, and P. Zurita-Gotor, 2006: A gray-radiation aquaplanet moist GCM. Part 1: Static stability and eddy scale. J. Atmos. Sci., 63, 2548-2566, https://doi.org/10.1175/ JAS3753.1.

Graversen, R. G., P. L. Langen, and T. Mauritsen, 2014: Polar amplification in CCSM4: Contributions from the lapse rate and the surface albedo feedbacks. J. Climate, 27, 4433-4450, https://doi.org/10.1175/JCLI-D-13-00551.1.

Hansen, J., and Coauthors, 2005: Efficacy of climate forcings. J. Geophys. Res., 110, D18104, https://doi.org/10.1029/ 2005JD005776.

Huang, Y., and M. Zhang, 2014: The implication of radiative forcing for poleward energy transport. Geophys. Res. Lett., 41, 1665-1672, https://doi.org/10.1002/2013GL059079.

_, X. Tan, and Y. Xia, 2016: Inhomogeneous radiative forcing of homogeneous greenhouse gases. J. Geophys. Res. Atmos., 121, 2780-2789, https://doi.org/10.1002/2015JD024569.

IPCC, 2013: Climate Change 2013: The Physical Science Basis. T. F. Stocker et al., Eds., Cambridge University Press, 1535 pp.

Langen, P. L., R. G. Graversen, and T. Mauritsen, 2012: Separation of contributions from radiative feedbacks to polar amplification on an aquaplanet. J. Climate, 25, 3010-3024, https:// doi.org/10.1175/JCLI-D-11-00246.1.

Lee, S., 2014: A theory for polar amplification from a general circulation perspective. Asia-Pac. J. Atmos. Sci., 50, 31-43, https://doi.org/10.1007/s13143-014-0024-7.

Manabe, S., and R. T. Wetherald, 1975: The effects of doubling the $\mathrm{CO}_{2}$ concentration on the climate of a general circulation model. J. Atmos. Sci., 32, 3-15, https://doi.org/10.1175/15200469(1975)032<0003:TEODTC $>2.0$. CO;2.

_- R. Stouffer, M. Spelman, and K. Bryan, 1991: Transient responses of a coupled ocean-atmosphere model to gradual changes of atmospheric $\mathrm{CO}_{2}$. Part I. Annual mean response. J. Climate, 4, 785-818, https://doi.org/10.1175/ 1520-0442(1991)004<0785:TROACO >2.0.CO;2.

Mauritsen, T., R. G. Graversen, D. Klocke, P. L. Langen, B. Stevens, and L. Tomassini, 2013: Climate feedback efficiency and synergy. Climate Dyn., xx, 2539-2554, https:// doi.org/10.1007/s00382-013-1808-7.

Merlis, T. M., 2014: Interacting components of the top-of-atmosphere energy balance affect changes in regional surface temperature. Geophys. Res. Lett., 41, 7291-7297, https://doi.org/10.1002/ 2014GL061700.

, 2015: Direct weakening of tropical circulations from masked $\mathrm{CO}_{2}$ radiative forcing. Proc. Natl. Acad. Sci. USA, 112, 13167 13171, https://doi.org/10.1073/pnas.1508268112.

_ and M. Henry, 2018: Simple estimates of polar amplification in moist diffusive energy balance models. J. Climate, 31, 58115824, https://doi.org/10.1175/JCLI-D-17-0578.1.

O'Gorman, P. A., and T. Schneider, 2008: The hydrological cycle over a wide range of climates simulated with an idealized GCM. J. Climate, 21, 3815-3832, https://doi.org/10.1175/ 2007JCLI2065.1.

, and M. S. Singh, 2013: Vertical structure of warming consistent with an upward shift in the middle and upper troposphere. Geophys. Res. Lett., 40, 1838-1842, https://doi.org/10.1002/grl.50328. 
Payne, A. E., M. F. Jansen, and T. W. Cronin, 2015: Conceptual model analysis of the influence of temperature feedbacks on polar amplification. Geophys. Res. Lett., 42, 9561-9570, https:// doi.org/10.1002/2015GL065889.

Pithan, F., and T. Mauritsen, 2014: Arctic amplification dominated by temperature feedbacks in contemporary climate models. Nat. Geosci., 7, 181-184, https://doi.org/10.1038/ ngeo2071.

Po-Chedley, S., and Q. Fu, 2012: Discrepancies in tropical upper tropospheric warming between atmospheric circulation models and satellites. Environ. Res. Lett., 7, 044018, https://doi.org/ 10.1088/1748-9326/7/4/044018.

Roe, G. H., and M. B. Baker, 2007: Why is climate sensitivity so unpredictable? Science, 318, 629-632, https://doi.org/10.1126/ science. 1144735.

Santer, B. D., and Coauthors, 2005: Amplification of surface temperature trends and variability in the tropical atmosphere. Science, 309, 1551-1556, https://doi.org/10.1126/ science. 1114867.
Smith, K. L., G. Chiodo, M. Previdi, and L. M. Polvani, 2018: No surface cooling over Antarctica from the negative greenhouse effect associated with instantaneous quadrupling of $\mathrm{CO}_{2}$ concentrations. J. Climate, 31, 317-323, https://doi.org/10.1175/ JCLI-D-17-0418.1.

Soden, B. J., I. M. Held, R. Colman, K. M. Shell, J. T. Kiehl, and C. A. Shields, 2008: Quantifying climate feedbacks using radiative kernels. J. Climate, 21, 3504-3520, https://doi.org/ 10.1175/2007JCLI2110.1.

Weaver, C., and V. Ramanathan, 1995: Deductions from a simple climate model: Factors governing surface temperature and atmospheric thermal structure. J. Geophys. Res., 100, $11585-$ 11 591, https://doi.org/10.1029/95JD00770.

Winton, M., 2006: Amplified Arctic climate change: What does surface albedo feedback have to do with it? Geophys. Res. Lett., 33, L03701, https://doi.org/10.1029/2005GL025244.

Zhang, M., and Y. Huang, 2014: Radiative forcing of quadrupling $\mathrm{CO}_{2}$. J. Climate, 27, 2496-2508, https://doi.org/10.1175/ JCLI-D-13-00535.1. 